\title{
PRESSÃO POSITIVA NA VENTILAÇÃO MECÂNICA INVASIVA E IMPLICAÇÕES RENAIS EM PACIENTES CRÍTICOS
}

\section{POSITIVE PRESSURE ON INVASIVE MECHANICAL VENTILATION AND RENAL IMPLICATIONS IN CRITICAL PATIENTS}

\section{PRESIÓN POSITIVA SOBRE VENTILACIÓN MECÁNICA INVASIVA E IMPLICACIONES RENALES EN PACIENTES CRÍTICOS}

Natália Vieira Araújo Cunha ${ }^{1}$, Breno de Sousa Santana ${ }^{2}$, Tayse Tâmara da Paixão Duarte ${ }^{3}$, Wellington Luiz de Lima ${ }^{4}$, Layse Farias $\mathrm{Nava}^{5}$, Marcia Cristina da Silva Magro ${ }^{6}$

\section{RESUMO}

Objetivo: Verificar se há influência da ventilação mecânica com pressão positiva, ao final da expiração, na função renal de pacientes internados em unidade de terapia intensiva. Método: Estudo prospectivo e quantitativo realizado, em unidade de terapia intensiva, especializada em trauma ortopédico e medular. A amostra foi não probabilística, composta por 31 pacientes em ventilação mecânica invasiva que desenvolveram lesão renal aguda na internação. Os pacientes foram alocados em grupos, conforme valor da pressão positiva, ao final da expiração, e os dados coletados por questionário estruturado. Para a análise das variáveis, realizaram-se testes não paramétricos. Resultados com $p \leq 0,05$ foram considerados significativos. Resultados: A idade média foi $46,94 \pm 24,2$ anos com predominância do sexo masculino (61,3\%). A disfunção renal (70,97\%), no estágio 1 (risco) (35,5\%), predominou. Pacientes com maior pressão positiva, ao final da expiração, evoluíram com disfunção renal $(p=0,04)$. Além disso, para aqueles com pressão positiva, ao final da expiração $\geq 10 \mathrm{cmH} 20$, a idade $(p=0,05)$ e a disfunção renal $(p=0,04)$ mostraram-se associados significativamente. Conclusão: Pacientes críticos em ventilação mecânica invasiva com pressão positiva, ao final da expiração mais elevada, mostraram reunir maior predisposição para a lesão renal aguda.

Descritores: Lesão Renal Aguda; Respiração Artificial; Unidades de Terapia Intensiva.

\section{ABSTRACT}

Objective: To verify if there is influence of positive end-expiratory pressure (PEEP) used in invasive mechanical ventilation (IMV) on renal function in ICU patients. Method: Prospective and quantitative study conducted in an intensive care unit specialized in orthopedic and spinal cord trauma. The sample was non-probabilistic, consisting of 31 patients on invasive mechanical ventilation who developed acute kidney injury during hospitalization. Patients were allocated to groups according to positive end-expiratory pressure and data collected by a structured questionnaire. For the analysis of the variables, nonparametric tests were performed using SPSS software (version 23). Results with $p \leq 0.05$ were considered significant. Results: The mean age was $46.94 \pm 24.2$ years old, with male predominance (61.3\%). Renal dysfunction (70.97\%) in stage 1 (risk) (35.5\%) predominated. Patients with higher positive end-expiratory pressure developed renal dysfunction $(p=0.04)$. In addition for those with positive end-expiratory pressure $\geq 10 \mathrm{cmH} 2 \mathrm{O}$, age $(p=0.05)$ and renal dysfunction $(p=0.04)$ were significantly associated. Conclusion: Critical patients on invasive mechanical ventilation with positive end-expiratory pressure were shown to have a greater predisposition to acute kidney injury. Descriptors: Acute Kidney Injury; Respiration, Artificial; Intensive Care Units.

\section{RESUMEN}

Objetivo: Para verificar si hay influencia de la presión positiva al final de la espiración (PEEP) utilizada en la ventilación mecánica invasiva (IMV) en la función renal en pacientes de la UCI. Método: Estudio prospectivo y cuantitativo realizado en una unidad de cuidados intensivos especializada en traumatismos ortopédicos y de médula espinal. La muestra no fue probabilística, consistió en 31 pacientes con ventilación mecánica invasiva que desarrollaron lesión renal aguda durante la hospitalización. Los pacientes fueron asignados a grupos de acuerdo con la presión positiva al final de la espiración y los datos recopilados mediante un cuestionario estructurado. Para el análisis de variables, se realizaron pruebas no paramétricas en SPSS versión 23. Los resultados con $p \leq 0.05$ se consideraron significativos. Resultados: La edad media fue de 46,94 $\pm 24,2$ años con mayoria masculina (61,3\%). Predominó la disfunción renal (70.97\%) en la etapa 1 (riesgo) (35.5\%). Los pacientes con mayor presión positiva al final de la espiración desarrollaron disfunción renal $(p=0.04)$. Además, para aquellos con presión positiva al final de la espiración $\geq 10 \mathrm{cmH} 20$, la edad $(p=0.05)$ y la disfunción renal $(p=0.04)$ se asociaron significativamente. Conclusion: Los pacientes críticos con ventilación mecánica invasiva y con presión positiva al final de la espiración mostraron una mayor predisposición a la lesión renal aguda. Descriptores: Lesión Renal Aguda; Respiración Artificial; Unidades de Cuidados Intensivos.

${ }^{1}$ Enfermeira. Mestre em Enfermagem pela Universidade de Brasília. ${ }^{2}$ Enfermeiro. Especialista em Enfermagem, Universidade de Brasília. ${ }^{3}$ Enfermeira. Mestre em Enfermagem pela Universidade de Brasília. ${ }^{4}$ Enfermeiro. Especialista em Enfermagem, Universidade de Brasília. ${ }^{5}$ Enfermeira pela Universidade de Brasília. ${ }^{6}$ Enfermeira. Doutora pela Universidade de São Paulo, Docente na Universidade de Brasília. 


\section{INTRODUÇÃO}

A pressão positiva, ao final da expiração (PEEP), na ventilação mecânica invasiva (VMI), pode provocar alterações neuro-hormonais em pacientes críticos, decorrentes da ativação do sistema neurovegetativo simpático, reninaangiotensina e supressão da liberação do peptídeo natriurético atrial, induzindo a retenção de líquidos e a diminuição do fluxo sanguíneo renal, potenciais causas de lesão renal aguda $(\text { LRA })^{(1)}$. Sendo assim, a identificação de mecanismos e/ou de fatores modificáveis pode ser útil, para melhor direcionar a gestão, além do processo de sistematização da assistência e segurança do paciente crítico com LRA $^{(1-2)}$.

A ventilação mecânica invasiva associada a altas pressões intratorácicas, resultante da interação ventilador-pulmão, pode reduzir o débito cardíaco, predispondo a perfusão renal inadequada, anormalidades nas trocas gasosas determinantes da hipoxemia, hipercarbia e acidose sistêmica que, por sua vez, podem influenciar a resistência vascular renal, alterando as pressões de perfusão renal, culminando em $\operatorname{LRA}^{(3)}$.

A LRA é uma síndrome, potencialmente reversível definida como redução abrupta da função renal associada ao acúmulo de solutos nitrogenados. Constata-se, por meio da avaliação do nível sérico da creatinina e do débito urinário, em um período de até sete dias, com potencial reversibilidade em um intervalo de 48 horas após início do insulto ${ }^{(4)}$.

Verifica-se que a etiologia da LRA é multifatorial ${ }^{(5)}$ e sua incidência mundial permanece elevada, em pacientes críticos de unidades de terapia intensiva (UTI) (35\% a 45\%), quando comparada à unidade de internação (10\% a $18 \%)$, sobretudo, em países em desenvolvimento. Estudos têm mostrado associação entre a ocorrência de LRA e VMI no Brasil e no mundo ${ }^{(6-8)}$. A relação entre pulmões e rins é clinicamente importante, no processo saúde-doença, considerando que a VMI provoca anormalidades hemodinâmicas associadas à redução do débito cardíaco, comprometendo a perfusão renal, bem como a estimulação hormonal e as vias simpáticas ${ }^{(8-9)}$.

A previsão precoce do risco de complicações e mortalidade, para pacientes com LRA, pode contribuir e direcionar tomadas de decisão, em tempo de ações apropriadas serem assumidas, considerando que a LRA é uma doença heterogênea e sua causa é complexa, o que torna essas previsões uma tarefa desafiadora ${ }^{(10)}$. A LRA é, portanto uma condição que os países em desenvolvimento não podem ignorar, e a apreciação de seu padrão local pode contribuir e fundamentar políticas para a sua prevenção e gestão ${ }^{(11)}$. Quanto à importância desta temática, o objetivo deste estudo foi verificar se há influência da pressão positiva, ao final da expiração (PEEP) utilizada, na ventilação mecânica invasiva (VMI), na função renal de pacientes internados em UTI.

\section{MÉTODOS}

Estudo observacional, longitudinal, prospectivo e quantitativo, realizado na Unidade de Terapia Intensiva, especializada em trauma ortopédico e medular de um hospital da rede pública do Distrito Federal, Brasil, no período de dezembro de 2015 a dezembro de 2016.

A amostra foi não probabilística (conveniência), constituída de 31 pacientes internados na UTI por, pelo menos, sete dias e, ao término desse período, verificou-se o desfecho (LRA e óbito).

Os critérios de inclusão foram pacientes com idade igual ou superior a 18 anos, com alteração da creatinina sérica por período superior a 24 horas após a internação na UTI. Foram excluídos pacientes com história de lesão renal prévia, segundo registros no prontuário eletrônico e insuficiência renal crônica (estágios 4 e 5) pela classificação Kidney Disease Improving Global Outcomes (KDIGO) ${ }^{(4)}$.

O nível sérico da creatinina basal foi aquele registrado até um mês antes da internação na UTI. Para a coleta de dados, adotou-se um questionário constituído de dados demográficos dos pacientes, história clínica (comorbidades, tempo de internação, tempo de ventilação mecânica, parâmetros ventilatórios), variáveis hemodinâmicas (pressão arterial, oximetria, frequência respiratória, frequência cardíaca, temperatura), parâmetros laboratoriais (creatinina, potássio e ureia séricos) registrados diariamente.

Para a avaliação dos parâmetros laboratoriais, adotou-se o valor de referência assumido pela instituição hospitalar do estudo: Creatinina (mg/dL): 0,80 - 1,40; Potássio $(\mathrm{mEq} / \mathrm{L})$ : 3,60 - 5,0; Sódio (mEq/L): 135,0 - 148,0; Ureia (mg/dL): 10,0 - 50,0; Hemoglobina (g/dL): 13,0 17,0; e Hematócrito (\%): 42,0 - 50,0.

A coleta de dados obedeceu ao protocolo:

Etapa 1: alocação do paciente, em um determinado grupo, de acordo com o valor da PEEP programada no ventilador mecânico, a saber: (1) grupo 1 - VMI com PEEP $\leq 5 \mathrm{cmH} 2 \mathrm{O}$; (2) 
grupo 2 - VMI com PEEP > 5cmH2O e < $10 \mathrm{cmH} 2 \mathrm{O}$; (3) grupo $3-\mathrm{VMI}$ com PEEP $\geq$ $10 \mathrm{cmH} 2 \mathrm{O}$.

Etapa 2: acompanhamento diário do paciente, durante sete dias, por meio de registros do prontuário.

Etapa 3: estadiamento da gravidade do comprometimento da função renal, a partir dos valores de creatinina sérica, conforme a classificação KDIGO(4).

Etapa 4: análise do desfecho dos participantes, ao término da internação na UTI (alta, óbito, transferência).

A avaliação da função renal foi fundamentada na classificação $\mathrm{KDIGO}^{(4)}$ - que permite o estadiamento da função renal, a partir da variação do valor da creatinina sérica, em relação à creatinina basal e ao débito urinário. $A$ severidade do comprometimento é determinada por estágios distribuídos da seguinte forma: (1) estágio 1 (menor gravidade), quando há aumento de 1,5 a 1,9 vezes ou $0,3 \mathrm{mg} / \mathrm{dL}$ na creatinina basal e débito urinário menor que $0,5 \mathrm{~mL} / \mathrm{kg} / \mathrm{h}$ por 6 a 12 horas; (2) estágio 2 (gravidade moderada), quando há aumento de 2,0 a 2,9 vezes na creatinina basal ou débito urinário menor que $0,5 \mathrm{~mL} / \mathrm{kg} / \mathrm{h}$ por tempo maior ou igual 12 horas e, por fim (3), o estágio 3 (maior gravidade), configura-se pelo aumento de 3,0 vezes na creatinina basal ou aumento de 4,0 $\mathrm{mg} / \mathrm{dL}$ ou início de terapia dialítica, em menores de 18 anos, com o clearance de creatinina inferior a $35 \mathrm{~mL} / \mathrm{min} / 1,73 \mathrm{~m}^{2}$ e/ou débito urinário menor que $0,3 \mathrm{~mL} / \mathrm{kg} / \mathrm{h}$ por tempo maior ou igual a 24 horas ou anúria por tempo igual ou maior a 12 horas.

Os dados foram expressos em frequência absoluta (n), frequência relativa (\%), média e desvio-padrão para a distribuição normal da amostra. Para a distribuição assimétrica, verificada após teste de normalidade, calcularam-se a mediana e o percentil 25 e 75 e aplicaram-se os testes não paramétricos Exato de Fisher e MannWhitney. Foram considerados significativos os resultados com valores de $p \leq 0,05$.

O consentimento foi obtido por meio do paciente ou seu responsável legal. Este estudo foi aprovado pelo Comitê de Ética em Pesquisa da Fundação de Ensino e Pesquisa em Ciências da Saúde (CEP/FEPECS) sob CAEE 18021313.5.0000.5553.

\section{RESULTADOS E DISCUSSÃO}

Do total de 31 pacientes, a maioria era do sexo masculino $(61,3 \%)$ com idade e índice de massa corporal medianos de 43 (27-64) anos e 23 (21-25) $\mathrm{kg} / \mathrm{m} 2$, respectivamente. O tempo de internação foi de $10,5 \pm 37,2$ dias. A maioria fez uso de antibióticos $(96,8 \%)$, noradrenalina $(83,9 \%)$ e furosemida $(67,7 \%)$. A mortalidade acometeu $9,7 \%$ dos pacientes (Tabela 1 ).

Tabela 1 - Distribuição dos pacientes $(n=31)$ segundo as características sociodemográficas e clínicas. Brasília, DF, Brasil, 2016.

\begin{tabular}{|c|c|c|c|}
\hline Variável & n (\%) & Média $\pm D P$ & Mediana (25-75) \\
\hline \multicolumn{4}{|l|}{ Sexo } \\
\hline Masculino & $19(61,3)$ & - & - \\
\hline Feminino & $12(38,7)$ & - & - \\
\hline Idade & - & $46,94 \pm 24,2$ & $43(27-64)$ \\
\hline Altura & - & $164,3 \pm 9,7$ & $164(155-172)$ \\
\hline IMC & - & $23,5 \pm 4,0$ & $23(21-25)$ \\
\hline Peso & - & $63,1 \pm 10,3$ & 59 (57-70) \\
\hline Óbito & $3(9,7)$ & - & - \\
\hline \multicolumn{4}{|l|}{ Número de dias } \\
\hline Internação & - & $10,5 \pm 37,2$ & \\
\hline VMI & - & $22,0 \pm 55$ & $7(7-11)$ \\
\hline
\end{tabular}




\begin{tabular}{|c|c|c|c|}
\hline Variável & n (\%) & Média $\pm D P$ & $\begin{array}{c}\text { Mediana } \\
(25-75)\end{array}$ \\
\hline \multicolumn{4}{|l|}{ Número de dias } \\
\hline Noradrenalina & - & $4,0 \pm 2,5$ & - \\
\hline Furosemida & - & $3,0 \pm 2,8$ & - \\
\hline Antibiótico & - & $5,7 \pm 2,0$ & - \\
\hline Sonda vesical de demora & - & $6,0 \pm 2,0$ & - \\
\hline \multicolumn{4}{|l|}{ Uso de medicações } \\
\hline Noradrenalina & $26(83,9)$ & - & - \\
\hline Dobutamina & $2(6,4)$ & - & - \\
\hline Furosemida & $21(67,7)$ & - & - \\
\hline Antibiótico & $30(96,8)$ & - & - \\
\hline
\end{tabular}

IMC= Índice de Massa Corporal. VMI= Ventilação Mecânica Invasiva.

Os resultados mostraram preservação do perfil hemodinâmico e algumas alterações de variáveis biológicas, como elevação sérica da ureia $(59,3 \pm 35 \mathrm{mg} / \mathrm{dL})$, redução da hemoglobina
$(10,7 \pm 9,1 \mathrm{~g} / \mathrm{dL})$ e do hematócrito $(31,3 \pm 6,6 \%)$. Houve tendência à manutenção de $\mathrm{pH}$ sanguíneo ácido $(7,3 \pm 0,09)$ (Tabela 2$)$.

Tabela 2 - Distribuição das variáveis hemodinâmicas e biológicas dos pacientes $(n=31)$ internados na Unidade de Terapia Intensiva. Brasília, DF, Brasil, 2016.

\begin{tabular}{|c|c|}
\hline Variável & Média $\pm D P$ \\
\hline \multicolumn{2}{|l|}{ Hemodinâmica } \\
\hline PAS (mmHg) & $133,0 \pm 23$ \\
\hline $\mathrm{PAD}(\mathrm{mmHg})$ & $70,0 \pm 16$ \\
\hline $\mathrm{FC}(\mathrm{bpm})$ & $85 \pm 22$ \\
\hline FR (rpm) & $17 \pm 3$ \\
\hline $\mathrm{SpO} 2(\%)$ & $97 \pm 7$ \\
\hline \multicolumn{2}{|l|}{ Biológica } \\
\hline Creatinina (mg/dL) & $1,05 \pm 0,6$ \\
\hline Potássio (mEq/L) & $3,8 \pm 0,7$ \\
\hline Sódio (mEq/L) & $140 \pm 11$ \\
\hline Ureia (mg/dL) & $59,3 \pm 35$ \\
\hline Hemoglobina (g/dL) & $10,7 \pm 9,1$ \\
\hline Hematócrito (\%) & $31,3 \pm 6,6$ \\
\hline $\mathrm{pH}$ & $7,3 \pm 0,09$ \\
\hline
\end{tabular}

PAS=pressão arterial sistólica. $\mathrm{PAD}=$ pressão arterial diastólica. $\mathrm{FC}=$ frequência cardíaca. $\mathrm{FR}=$ frequência respiratória. $\mathrm{SpO} 2=$ saturação periférica de oxigênio. 
A maioria dos pacientes, em ventilação mecânica invasiva com pressão positiva, evoluíram com disfunção renal estágio 1 , ou seja, de menor gravidade $(35,5 \%)$ e um menor percentual no estágio 2 , de moderada gravidade (16,1\%), conforme classificação KDIGO (Tabela 3).

Tabela 3 - Distribuição dos pacientes ( $n=31$ ), em estágios de disfunção renal, de acordo com a classificação KDIGO. Brasília, DF, Brasil, 2016.

\begin{tabular}{lcc}
\hline \hline & Estágio & $\mathbf{n}(\%)$ \\
\hline Estágio 1 (risco) & $11(35,5)$ \\
Estágio 2 (lesão) & $5(16,1)$ \\
Estágio 3 (falência) & $6(19,4)$ \\
\hline \hline
\end{tabular}

Houve uma tendência de pacientes com insuficiência respiratória aguda (IRpA) $(31,8 \%)$ e pneumonia (50\%) evoluírem mais frequentemente à disfunção renal. Pacientes com

Tabela 4 - Análise univariada das características clínicas, em relação à função renal dos participantes internados, na Unidade de Terapia Intensiva. Brasília, DF, Brasil, 2016.

\begin{tabular}{|c|c|c|c|c|c|}
\hline \multirow[t]{2}{*}{ Características } & \multicolumn{2}{|c|}{ Sem disfunção renal $(n=9)$} & \multicolumn{2}{|c|}{ Com disfunção renal $(n=22)$} & \multirow[t]{2}{*}{$\mathbf{p}$} \\
\hline & n (\%) & Mediana (25-75) & n (\%) & Mediana (25-75) & \\
\hline Sexo masculino & $5(55,6)$ & & $14(63,6)$ & & $0,5^{\ddagger}$ \\
\hline Idade (anos) & & $35(31-51)$ & & $46(25-67)$ & $0,7^{+}$ \\
\hline $\mathrm{IMC}\left(\mathrm{kg} / \mathrm{m}^{2}\right)$ & & $24,2(22,1-25,6)$ & & $22,5(20,0-23,9)$ & $0,2^{\dagger}$ \\
\hline Tempo de noradrenalina (dias) & & $4(3-6)$ & & $5(4-7)$ & $0,3^{+}$ \\
\hline Tempo de furosemida (dias) & & $2(1-6)$ & & $4(4-7)$ & $0,2^{+}$ \\
\hline Tempo de ATB (dias) & & $7(3-7)$ & & $7(6-7)$ & $0,5^{\dagger}$ \\
\hline Tempo de VM (dias) & & $7(5-7)$ & & $7(7-12)$ & $0,3^{+}$ \\
\hline Óbito & $1(11,1)$ & & $2(9,1)$ & & $0,7^{\ddagger}$ \\
\hline IRpA & $5(55,6)$ & & $11(50,0)$ & & $0,5^{\ddagger}$ \\
\hline PEEP - Grupo 2 & $9(100,0)$ & & $14(63,6)$ & & $0,04^{\ddagger}$ \\
\hline PEEP - Grupo 3 & $0(0,0)$ & & $8(36,4)$ & & $0,04^{\ddagger}$ \\
\hline \multicolumn{6}{|l|}{ Diagnóstico atual } \\
\hline Choque séptico & $2(22,2)$ & & $5(22,7)$ & & $0,7^{\ddagger}$ \\
\hline Pneumonia & $5(55,6)$ & & $11(50,0)$ & & $0,5^{\ddagger}$ \\
\hline Sepse origem pulmonar & $2(22,2)$ & & $2(9,1)$ & & $0,3^{\ddagger}$ \\
\hline IRpA & $5(55,6)$ & & $7(31,8)$ & & $0,2^{\ddagger}$ \\
\hline POI de fratura transtrocantérica & $1(11,1)$ & & $6(27,3)$ & & $0,3^{\ddagger}$ \\
\hline \multicolumn{6}{|l|}{ Comorbidades } \\
\hline HAS & $2(22,2)$ & & $3(13,6)$ & & $0,5^{\ddagger}$ \\
\hline DM & $2(22,2)$ & & $2(9,1)$ & & $0,3^{\ddagger}$ \\
\hline
\end{tabular}

PEEPs mais elevadas evoluíram com disfunção renal $(p=0,04)$. Não houve pacientes classificados no Grupo 1 (PEEP $\leq 5 \mathrm{~cm} / \mathrm{H} 2 \mathrm{O}$ ) (Tabela 4). 


\section{Drogas em uso}

Noradrenalina

$19(86,4)$

$0,5^{\ddagger}$

Furosemida

$5(55,6)$

$16(72,7)$

$0,3^{\ddagger}$

† Teste de Mann-Whitney; $¥$ Teste de Fisher; IMC= índice de massa corporal; $A T B=$ antibiótico; VM= ventilação mecânica; IRpA= insuficiência respiratória aguda; $P E E P=$ pressão positiva no final da expiração; Grupo PEEP 2= PEEP>5 e $\leq 10 \mathrm{cmH} 2 \mathrm{O}$; Grupo PEEP 3$\mathrm{PEEP}>10 \mathrm{cmH} 2 \mathrm{O}$; $\mathrm{POI}=$ pós-operatório imediato; $\mathrm{HAS}=$ hipertensão arterial sistêmica; $\mathrm{DM}=$ diabetes mellitus.

Possuir idade mediana mais jovem [27 (22 - 38) anos] interferiu, significativamente, para a necessidade de uso de ventilação mecânica com PEEP mais elevada $(>10 \mathrm{cmH} 2 \mathrm{O})(\mathrm{p}=0,05)$. A obesidade e o sexo não influenciaram o valor da
PEEP empregada na estratégia ventilatória ( $p>0,05)$. Os pacientes com PEEP $>5$ e $\leq 10 \mathrm{cmH} 20$ evoluíram mais frequentemente ao óbito (13\%), embora sem significância estatística (Tabela 5).

Tabela 5 - Relação entre as características clínicas e valor da pressão positiva, ao final da expiração (PEEP) dos participantes internados, na Unidade de Terapia Intensiva. Brasília, DF, Brasil, 2016.

\begin{tabular}{|c|c|c|c|c|c|}
\hline \multirow[t]{2}{*}{ Características } & \multicolumn{2}{|c|}{$\begin{array}{l}\text { PEEP - Grupo } 2 \\
\quad(n=23)\end{array}$} & \multicolumn{2}{|c|}{$\begin{array}{l}\text { PEEP - Grupo } 3 \\
(n=8)\end{array}$} & \multirow[t]{2}{*}{ p } \\
\hline & $n(\%)$ & Mediana (25-75) & $n(\%)$ & Mediana (25-75) & \\
\hline Sexo masculino & $13(56,5)$ & & $6(75,0)$ & & $0,3^{\ddagger}$ \\
\hline Idade (anos) & & $49(34-66)$ & & $27(22-38)$ & $0,05^{\dagger}$ \\
\hline $\mathrm{IMC}\left(\mathrm{kg} / \mathrm{m}^{2}\right)$ & & $23,6(21,5-25,8)$ & & $22,2(19,6-23,9)$ & $0,4^{\dagger}$ \\
\hline $\begin{array}{l}\text { Tempo de noradrenalina } \\
\text { (dias) }\end{array}$ & & $5(4-7)$ & & $6(3-6,5)$ & $0,9^{\dagger}$ \\
\hline Tempo de furosemida (dias) & & $4(3,5-6,5)$ & & $5(3-7)$ & $0,8^{+}$ \\
\hline Tempo de ATB (dias) & & $7(5-7)$ & & $7(5-7)$ & $0,8^{+}$ \\
\hline Tempo de VM (dias) & & $7(5-7)$ & & $7(7-12)$ & $0,7^{+}$ \\
\hline Óbito & $3(13,0)$ & & $0(0,0)$ & & $0,4^{\ddagger}$ \\
\hline \multicolumn{6}{|l|}{ História clínica } \\
\hline Trauma raquimedular & $7(30,4)$ & & $3(37,5)$ & & $0,5^{\ddagger}$ \\
\hline Pneumonia não espec. & $13(56,6)$ & & $3(37,5)$ & & $0,3^{\ddagger}$ \\
\hline Dispneia & $6(26,1)$ & & $1(12,5)$ & & $0,4^{\ddagger}$ \\
\hline IRpA & $13(56,5)$ & & $3(37,5)$ & & $0,3^{\ddagger}$ \\
\hline Disfunção renal & $14(60,9)$ & & $8(100,0)$ & & $0,04^{\ddagger}$ \\
\hline \multicolumn{6}{|l|}{ Comorbidades } \\
\hline HAS & $5(21,7)$ & & $0(0,0)$ & & $0,2^{\ddagger}$ \\
\hline DM & $3(13,0)$ & & $1(12,5)$ & & $0,7^{\ddagger}$ \\
\hline \multicolumn{6}{|l|}{ Drogas em uso } \\
\hline Noradrenalina & $19(82,6)$ & & $7(87,5)$ & & $0,6^{\ddagger}$ \\
\hline Furosemida & $16(69,6)$ & & $5(62,5)$ & & $0,5^{\ddagger}$ \\
\hline
\end{tabular}

† Teste de Mann-Whitney; $¥$ Teste de Fisher; IMC= índice de massa corporal; $A T B=$ antibiótico; VM= ventilação mecânica; IRpA= insuficiência respiratória aguda; HAS= hipertensão arterial sistêmica; DM= diabetes mellitus. 
A VMI é usualmente considerada como fator de risco para LRA em pacientes críticos. Os resultados deste estudo mostraram que $O$ emprego da PEEP, com valores mais elevados, durante a VMI, tende induzir a LRA $(p=0,04)$. Essa relação pode ser atribuída a fatores hemodinâmicos, que, quando alterados, influenciam a perfusão renal determinando a vasoconstrição, condição que predispõe à redução do débito urinário, da excreção urinária de sódio e da depuração de creatinina ${ }^{(8)}$.

A PEEP, enquanto pressão positiva, estabelecida a partir da VMI, provoca aumento da pressão intratorácica e, em consequência, alteração da função hemodinâmica, redução da resistência vascular sistêmica, compressão das veias cava superior e inferior, diminuição do enchimento ventricular, assim como do átrio esquerdo e, finalmente, redução do débito cardíaco e da pressão arterial ${ }^{(12)}$. A instabilidade hemodinâmica foi uma condição identificada, no presente estudo, pela necessidade de noradrenalina, droga vasoconstritora que promove elevação da pressão $\operatorname{arterial}^{(13)}$, no grupo de pacientes em VMI com elevação da PEEP.

Em pacientes de terapia intensiva, é comum a instabilidade dos sistemas fisiológicos, reconhecida pelas flutuações de pressão arterial, condições determinantes da necessidade de drogas vasoativas. Observa-se que essas drogas agem diretamente no sistema cardiovascular, sendo a noradrenalina, muitas vezes, selecionada e administrada pela sua ação agonista adrenérgica, responsável pela elevação e estabilização da pressão arterial ${ }^{(13)}$.

A estratégia ventilatória invasiva é um recurso adotado, para a recuperação do padrão respiratório, durante a hospitalização e o seu uso é considerado eficaz, no tratamento de diferentes patologias ${ }^{(14)}$, favorecendo a sobrevivência dos pacientes, por período mais prolongado, como identificado nesta investigação, em que a mortalidade acometeu $9,7 \%$ dos pacientes. Os indivíduos mais jovens, ainda, compuseram o grupo com PEEP mais elevada em comparação aos indivíduos mais velhos. A LRA é uma condição complexa subjacente a diferentes etiologias e mecanismos fisiopatológicos; comumente é diagnosticada em pacientes hospitalizados e contribui ao aumento da morbidade, mortalidade, assim como dos custos à saúde ${ }^{(15)}$ Biomarcadores foram adotados, para predizer os resultados da $\operatorname{LRA}^{(16-17)}$, no entanto a história clínica dos pacientes com traumatismo raquimedular fundamenta a compreensão da mortalidade, em um percentual relativamente baixo de LRA, predominantemente, em pacientes jovens do sexo masculino, quando comparados a um estudo desenvolvido na Espanha, com 279 pacientes que mostrou uma taxa de mortalidade de $22 \%{ }^{(18)}$. Grandes estudos retrospectivos de LRA também mostram mortalidade superior à identificada no presente estudo (14 e 36\%) ${ }^{(19)}$.

Nessa vertente, foi possível identificar que até mesmo características demográficas, como o sexo, podem influenciar a ocorrência da LRA em pacientes em VMI. O sexo masculino foi predominante tanto neste como em outro estudo $^{(20)}$, ratificando a existência da proteção hormonal do sexo feminino, descrita pelo menor risco de inibição do estrogênio e ativação de andrógenos ${ }^{(21)}$. Destaca-se que o estrogênio afeta o rim pela diminuição da proliferação de células mesangiais, aumento da atividade das enzimas metaloproteinases e síntese de óxido nítrico, alterações na inflamação, diminuição da atividade do sistema renina-angiotensina. Ressalta-se, na doença renal, a presença de outros mecanismos intervenientes, diferentes do sexo, como a hemodinâmica renal, a dieta, o tamanho renal/glomerular e os polimorfismos genéticos $^{(22)}$.

No contexto clínico do paciente crítico, o emprego de diuréticos tem sido associado ao maior risco de LRA. A teoria da preservação da oxigenação medular renal induzida por diuréticos, para prevenir LRA, não foi comprovada. É controversa a questão da diurese euvolêmica forçada/provocada para prevenir a LRA. Os diuréticos não diminuem a duração dessa síndrome e não reduzem a necessidade de terapia de substituição renal. Eles têm importância sobre a gestão volêmica na lesão renal $^{(23)}$. Os nossos achados sugerem que a maioria dos pacientes que necessitaram de furosemida tenderam evoluir com disfunção renal.

Assinala-se que a gestão da LRA ganhou singularidade, a partir do Kidney Disease Improving Global Guidelines (KDIGO) $)^{(4)}$, diretriz de práticas clínicas para LRA, que, neste contexto, quando adotada, permitiu a identificação predominante de disfunção renal de leve gravidade (estágio 1) na maioria dos pacientes. Além disso, embora inferior, um percentual também importante evoluiu no estágio de maior 
gravidade (falência renal). Estudo coorte retrospectivo, realizado em uma UTI do México, evidenciou que a maioria dos pacientes evoluiu no estágio 1 , seguidos pelos estágios 3 e 2, resultado similar aos achados no presente estudo $^{(24)}$.

As limitações podem ser descritas pelo fato de o estudo ter sido desenvolvido, em um único centro especializado em traumas ortopédico e medular, além do pequeno tamanho amostral. Vale destacar que estratégias eficazes de tratamento e prevenção da LRA podem ser possíveis no futuro, mas, por enquanto, os esforços devem se concentrar na identificação precoce de pacientes com LRA agravada e, no início de pacotes de cuidados projetados, para mitigar os fatores de risco modificáveis e fornecer cuidados de suporte intensivos na UTI. Estudos futuros devem concentrar-se na implantação de intervenções que utilizem pacotes de assistência, para LRA, com base em intervenções ativas de vigilância e fluxo de trabalho que forneçam suporte clínico à decisão para ajudar os profissionais de saúde a gerenciar essa síndrome de maneira mais eficaz. A capacidade de diagnosticar e detectar LRA, precocemente, pode reduzir o resultado negativo em pacientes em terapia intensiva.

\section{CONSIDERAÇÕES FINAIS}

Os resultados deste estudo mostraram que pacientes críticos em VMI com PEEP mais elevada reúnem maior predisposição para LRA. Além disso, a idade revelou-se como uma variável, que pode influenciar a determinação do valor de PEEP, durante a estratégia ventilatória.

\section{REFERÊNCIAS}

1. Muzaffar SN, Gurjar M, Baronia AK, Azim A, Mishra P, Poddar B, et al. Predictors and pattern of weaning and long-term outcome of patients with prolonged mechanical ventilation at an acute intensive care unit in North India. Rev Bras Ter Intensiva 2017;29(1):23-33. DOI: 10.5935/0103-507X.20170005

2. Kuiper JW, Groeneveld AJ, Haitsma JJ, Smeding L, Begieneman MP, Jothy $\mathrm{S}$, et al. Injurious mechanical ventilation causes kidney apoptosis and dysfunction during sepsis but not after intra-tracheal acid instillation: An experimental study. BMC Nephrol. 2014; 15:126. DOI: $10.1186 / 1471-2369-15-126$

3. Husain?Syed $\mathrm{F}$, Rosner $\mathrm{MH}$, Ronco $\mathrm{C}$. Distant organ dysfunction in acute kidney injury.
Acta Physiol 2019; 3:e13357. DOI: 10.1111/apha.13357

4. Kidney Disease Improving Global Outcomes (KDIGO). KDIGO clinical practice guideline for acute kidney injury. Kidney Int Suppl. 2012 [citado em 22 jan 2019]; 2(1):1-138. Disponível

em: https://linkinghub.elsevier.com/retrieve/pii/S215 $\underline{717161531073 \mathrm{X}}$

5. Flood L, Nichol A. Acute kidney injury and the critically ill. Anaesth Intensive Care Pain Med. 2018;19(3):113-8.

DOI: 10.1016/i.mpaic.2017.12.006

6. Santos LL, Magro MC S. Ventilação mecânica e a lesão renal aguda em pacientes na unidade de terapia intensiva. Acta Paul Enferm. 2015;28(2):146-51. DOI: $10.1590 / 1982-$ 0194201500025

7. Carneiro TA, Hermann PR S, Souza JMO, Magro MC S. Identification and recovery of renal function in non-dialytic patients in the intensive therapy scenario. Rer. Min. Enferm. 2017;21:1-6. DOI: $10.5935 / 1415-2762.20170039$

8. Cortes-Puentes GA, Oeckler RA, Marini JJ. Physiology-guided management of hemodynamics in acute respiratory distress syndrome. Ann Transl Med. 2018;6(18):353. DOI: 10.21037/atm.2018.04.40

9. Sriperumbuduri S, Clark E, Hiremath S. New insights into mechanisms of acute kidney injury in heart disease. Can J Cardiol. 2019;35(9):1158-69. DOI: 10.1016/j.cjca.2019.06.032

10. Xu Z, Luo Y, Adekkanattu P, Ancker JS, Jiang G, Kiefer RC, et al. Stratified mortality prediction of patients with Acute Kidney Injury in Critical Care. Stud Health Technol Inform. 2019;264:462-6. DOI: 10.3233/SHTI190264

11. Dlamini TAL, Heering PJ, Chivese $T$, Rayner B. A prospective study of the demographics, management and outcome of patients with acute kidney injury in Cape Town, South Africa. PLoS One 2017;12(6):e0177460. DOI: $10.1371 /$ journal.pone. 0177460

12. Barros AF, Barros LC, Sangean MC, Vega JM. Análise das alterações ventilatórias e hemodinâmicas com utilização de ventilação mecânica não-invasiva com binível pressórico em pacientes com insuficiência cardíaca congestiva. Arq Bras Cardiol. 2007;88(1):96-103. DOI: 10.1590/S0066-782X2007000100016

13. Melo EM, Oliveira TMM, Marques AM, Ferreira AMM, Silveira FMM, Lima VF. Caracterização dos pacientes em uso de drogas 
vasoativas internados em unidade de terapia intensiva. Rev Pesqui Cuid Fundam. 2016;8(3):4898-4904. DOI: $\quad 10.9789 / 2175-$ 5361.2016.v8i2.4898-4904

14. Loss SH, Oliveira RP, Maccari JG, Savi A, Boniatti MM, Hetzel MP, et al. The reality of patients requiring prolonged mechanical ventilation: A multicenter study. Rev Bras Ter Intensiva 2015;27(1):26-35. DOI: 10.5935/0103507X.20150006

15. Gameiro J, Agapito Fonseca J, Jorge S, Lopes J. Acute Kidney Injury Definition and Diagnosis: A narrative review. J Clin Med. 2018;7(10):307. DOI: 10.3390/jcm7100307

16. Wu V-C, Shiao C-C, Chi N-H, Wang C-H, Chueh S-CJ, Liou H-H, et al. Outcome prediction of Acute Kidney Injury biomarkers at initiation of dialysis in critical units. J Clin Med. 2018;7(8):202. DOI: $10.3390 / \mathrm{icm} 7080202$

17. Douvris A, Zeid K, Hiremath S, Brown P, Sood M, Arkoub RA, et al. Safety Lapses Prior to Initiation of Hemodialysis for Acute Kidney Injury in Hospitalized Patients: A Patient Safety Initiative. J Clin Med. 2018;7(10):317. DOI: $\underline{10.3390 / \mathrm{jcm} 7100317}$

18. Acosta-Ochoa I, Bustamante-Munguira J, Mendiluce-Herrero A, Bustamante-Bustamante J, Coca-Rojo A. Impact on outcomes across KDIGO2012 AKI criteria according to baseline renal function. J Clin Med. 2019;8(9):1323. DOI: 10.3390/jcm8091323

19. Hoste EAJ, Bagshaw SM, Bellomo R, Cely $\mathrm{CM}$, Colman R, Cruz DN, et al. Epidemiology of acute kidney injury in critically ill patients: The multinational AKI-EPI study. Intensive Care Med. 2015;41(8):1411-23. DOI: 10.1007/s00134-0153934-7

20. Peres LAB, Wandeur V, Matsuo T. Predictors of acute kidney injury and mortality in an Intensive Care Unit. J Bras Nefrol. 2015;37(1):38-46. DOI: 10.5935/01012800.20150007

21. Kang AK, Miller JA. Impact of gender on renal disease: The role of the renin angiotensin system. Clin Invest Med. 2003 [citado em 22 mar. 2019]; 26(1):38-44. Disponível em: http://www.ncbi.nlm.nih.gov/pubmed/12659469 22. Yu MK, Lyles CR, Bent-Shaw LA, Young BA. Risk factor, age and sex differences in chronic kidney disease prevalence in a diabetic cohort: The pathways study. Am J Nephrol. 2012;36(3):245-51. DOI: $10.1159 / 000342210$

23. Ejaz AA, Mohandas R. Are diuretics harmful in the management of acute kidney injury? Curr Opin Nephrol Hypertens. 2014;23(2):155-60. DOI: 10.1097/01.mnh.0000441150.17202.be

24. Córdova-Sánchez BM, Herrera-Gómez Á, Ñamendys-Silva SA. Acute Kidney Injury classified by serum creatinine and urine output in critically III cancer patients. Biomed Res Int. 2016;2016:17. DOI: $\underline{10.1155 / 2016 / 6805169}$

Nota: Este estudo foi derivado do projeto intitulado "Influência da ventilação mecânica sobre a função renal" e extraído do Trabalho de Conclusão de Residência da primeira autora. Este estudo não recebeu financiamento.

Recebido em: 10/07/2019

Aprovado em: 18/11/2019

Endereço de correspondência:

Breno de Sousa Santana

Campus Universitário - Centro Metropolitano, Ceilândia Sul. ZIP CODE: 72220-275 - Brasília/DF - Brasil

E-mail: bresousas@outlook.com 